\title{
Las tribulaciones de la madurez. Utilización correcta del NNT.
}

Molina M

\section{Hospital Infantil Universitario La Paz, Madrid.}

\section{Resumen}

El NNT se ideó valorar el efecto beneficioso de un tratamiento para disminuir el riesgo de que se produjese un evento desagradable en un grupo de intervención de interés, siempre respecto a lo observado en un grupo control. Se revisan algunos de los aspectos a tener en cuenta para utilizarlo de manera correcta.

\section{Introducción}

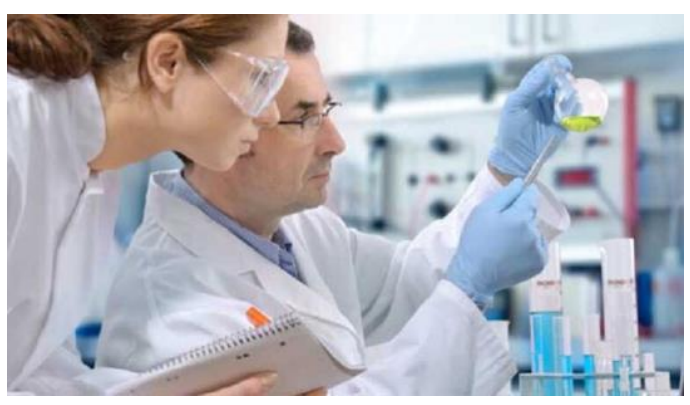

El NNT se ideó valorar el efecto beneficioso de un tratamiento para disminuir el riesgo de que se produjese un evento desagradable en un grupo de intervención de interés, siempre respecto a lo observado en un grupo control. Se revisan algunos de los aspectos a tener en cuenta para utilizarlo de manera correcta.

Érase una vez una época en la que los paradigmas científicos y la forma de pensar de los investigadores (y también de los clínicos) empezaron a cambiar, pasando del "me va bien" a querer saber realmente cuál era la validez de la información que recogían en sus experimentos o en su práctica diaria.
Es en este contexto cuando ve la luz, hace ya 33 años, el que vendría a convertirse en uno de los capos de las medidas de impacto de los estudios clínicos: el número necesario a tratar, mundialmente conocido por sus siglas, NNT.

\section{Número necesario a tratar (NNT)}

$\mathrm{Su}$ utilidad inicial fue valorar el efecto beneficioso de un tratamiento para disminuir el riesgo de que se produjese un evento desagradable en un grupo de intervención de interés, siempre respecto a lo observado en un grupo control. Dicho de forma más sencilla, surgió como medida de impacto en el contexto de los ensayos clínicos aleatorizados y controlados.

El NNT fue bien recibido inicialmente, ya que tiene el gran mérito de combinar, en un solo parámetro, los conceptos de significación estadística y de importancia clínica (siempre que se proporcione el valor de $\mathrm{p}$ o su intervalo de confianza). Además, es sencillo de interpretar para los clínicos sin necesidad de tener conocimientos profundos en estadística. 
Siguiendo un paralelismo con las edades de la vida, todo fue bien durante su infancia y siguió creciendo durante su juventud, extendiéndose a otros muchos ámbitos diferentes al ensayo clínico en paralelo convencional.

El problema de la juventud, además de estar al principio de la vida, es que dura poco. $\mathrm{Y}$ en la madurez, aunque sigue contando con seguidores entusiastas, el NNT ha empezado a acumular también detractores y críticos que han empezado a sacarle defectos.

De estas tribulaciones que sufre el NNT durante su madurez es de lo que vamos a hablar en esta entrada. Aunque hay quejas producidas por algunas de sus antipáticas características desde el punto de vista matemático por su herencia logarítmica, la mayoría se fundamentan, en realidad, en una mala comprensión de su significado o en un uso no del todo correcto del parámetro.

\section{Las tribulaciones del NNT}

Como ya hemos dicho, el NNT se desarrolló inicialmente para valorar la eficacia de un tratamiento para reducir el riesgo de producción de un suceso en un grupo de interés.

Por ejemplo si la mortalidad de la fildulastrosis es del 5\% anual y con un nuevo tratamiento baja al $3 \%$, querrá decir que el tratamiento disminuye el riesgo un $2 \%$, con lo que el NNT será de $1 / 0,02=50$. Esto significa que, por cada 50 pacientes que tratemos durante 1 año, evitaremos que uno se muera gracias al tratamiento. Eso sí, puede morirse si prolongamos el seguimiento, como luego veremos.

\section{La naturaleza dual del NNT}

Este enfoque es muy simplista, ya que el NNT, en realidad, tiene una naturaleza dual.
El NNT puede entenderse como el número necesario a tratar para conseguir aumentar en uno el número de sucesos positivos esperados o de disminuir en 1 el número de un suceso negativo, todo ello durante un periodo de seguimiento determinado.

Pero también se puede comprender en el sentido opuesto, en el de perjudicar: el número necesario a tratar para aumentar en 1 el número de un suceso negativo o para disminuir en 1 el número esperado de un suceso positivo, todo ello durante un periodo de seguimiento determinado.

Esto puede hacer que sea confuso valorar el NNT solo con su valor numérico. Para evitar este inconveniente, algunos autores han pensado que podemos llamar de forma distinta a cada una de las dualidades de NNT, de forma que siempre sabremos exactamente de qué estamos hablando.

En el fondo, estaríamos especificando la dirección del efecto que estudiamos.

Así, hablaríamos de número necesario a tratar para beneficiar (NNTB) cuando queramos expresar el número a tratar para conseguir un efecto beneficioso (o evitar uno desagradable), y de número necesario a dañar (NNTH, la "h" viene de harm, en inglés) cuando queramos referirnos al número necesario para que se produzca un efecto negativo (o se evite uno positivo que podría ocurrir sin el tratamiento).

\section{Intervalos imposibles}

Otro problema que molesta mucho a los detractores del NNT es que su cálculo puede, en ocasiones, generar valores negativos de su estimación puntual y, otras veces, intervalos de confianza que cruzan el cero hacia el reino de los números negativos. Aquí, la dificultad está en buscar un significado lógico a un NNT negativo. 
Imaginemos que obtenemos un NNT = 10 con un intervalo de confianza del 95\% (IC 95) de 8 a 12. Aquí no tenemos problema, tenemos que tratar a 10 (estimación puntual), aunque este valor puede ir desde 8 hasta 12 (estimación por intervalo).

El problema surge cuando aparecen números negativos. Por ejemplo, si el $\mathrm{NNT}=5$ con un IC 95 de 8 a -12 , ¿cómo lo valoramos?

Pues para esto nos viene bien recurrir a la naturaleza dual del NNT que hemos mencionado antes. Si lo pensamos bien, los valores de NNT entre -1 y 1 son imposibles. Así, el intervalo de 8 a -12 podríamos dividirlo en dos: NNTB de 8 (hasta infinito) y NNTH de 12 (hasta infinito). Pienso que la utilidad será escasa desde el punto de vista clínico pero, al menos, le habremos dado un sentido.

\section{Todo sería más fácil con una máquina del tiempo}

Como ya comentamos en una entrada anterior, cuando queremos estudiar la eficacia de una intervención, lo ideal sería dar el nuevo tratamiento, agotar el periodo de seguimiento y medir el efecto. Entonces usaríamos nuestra máquina del tiempo para volver al instante inicial $y$, en lugar del tratamiento en estudio, daríamos su alternativa, agotaríamos el seguimiento y mediríamos el resultado.

Una vez hecho esto, compararíamos los dos resultados. El problema, los más atentos ya os habréis dado cuenta, es que todavía no se ha inventado la máquina del tiempo. Esto hace que para obtener este otro resultado, que llamamos potencial o contrafactual, tengamos que recurrir al grupo de control de los ensayos, que nos sirve de sustituto.
$\mathrm{Si}$ pensamos un poco en las implicaciones de la teoría contrafactual, aunque la eficacia del tratamiento en estudio siempre sea la misma, el valor del NNT dependerá de con qué intervención lo comparemos. Por lo tanto, para interpretar correctamente el NNT siempre debe especificarse de forma explícita el comparador que hemos empleado.

Un NNT de 10 de un determinado tratamiento solo puede valorarse si se especifica cuál fue la intervención de control y cuáles fueron los tiempos de seguimiento, especialmente si son diferentes.

Así que id tomando nota: hay que expresar el valor del NNT junto con la alternativa de tratamiento y el periodo de seguimiento. No hacerlo así puede dificultar su valoración e inducir a error.

\section{Nunca desprecies un decimal}

Prácticamente todos los libros $\mathrm{y}$ manuales están de acuerdo en que el valor numérico obtenido para el NNT debe redondearse hasta el entero superior más próximo. Es lógico, no parece que tenga mucho sentido decir que tenemos que tratar a 4,8 pacientes, así que lo redondeamos a 5 .

El problema con el redondeo es que añade imprecisión a la estimación y puede inducir a error.

Por ejemplo, cualquiera de los valores de reducción absoluta de riesgo comprendidos entre 0,52 y 0,9 será equivalente, tras el redondeo a un NNT $=2$. Sin embargo, hay una gran diferencia entre una reducción del 52\% y una del $90 \%$. No deberíamos valorarla con el mismo NNT.

Entonces, si a nadie se le enciende el pelo cuando oye que la media es tener 1,2 hijos, ¿por qué esa fobia a dar 
decimales con el NNT? A fin de cuentas, no deja de ser un estimador que nosotros tenemos que saber interpretar.

Si vemos un NNT de 6,7 podemos concluir que tendríamos que tratar una media de 6 a 7 pacientes para conseguir un efecto beneficioso durante un periodo de tiempo determinado. Una advertencia, si lo hacemos así, habrá que dejar claro que la estimación estará entre 6 y 7 , pero que estos no son los límites del IC 95. No nos confundamos.

\section{No te olvides de las variables de confusión}

Ya sabéis que todos los estudios están sujetos al efecto de variables de confusión, sobre todo cuando no están aleatorizados. Estamos acostumbrados a ver las medidas de asociación ajustadas según las variables que los autores creen que pueden actuar como confusoras. Sin embargo, es frecuente ver que esto se olvida con el NNT y solo se proporciona su valor crudo.

Otras veces lo que ocurre es que se recurre a métodos de ajuste que no son apropiados. Se han desarrollado métodos muy diversos para calcular el NNT en múltiples escenarios, crudo y ajustado. Si no sabes cuál debe usar, busca a alguien que sepa antes de aplicar uno inadecuado.

\section{El tiempo también pasa para el NNT}

Ya lo hemos dicho antes, pero no está de más insistir: el valor del NNT depende de la longitud del periodo de seguimiento, así que debe especificarse. La proporción de eventos que se van produciendo va aumentando según va pasando el tiempo.

Por ejemplo, si el tratamiento produce una reducción del riesgo del evento que se mantiene constante en el tiempo, el valor del NNT irá siendo más bajo según aumenta la duración temporal del periodo de seguimiento. Se comprende fácilmente que hay que conocer la duración del seguimiento para interpretar correctamente el valor del NNT.

Pensemos dos ensayos clínicos de dos intervenciones diferentes, uno con un periodo de seguimiento de 2 años y el otro de 5. Aunque los dos estudios nos brindasen un NNT de 8 , no sería lo mismo tratarse 2 años que 5 para evitar un suceso desagradable por cada 8 pacientes tratados.

\section{Cuidado con los estudios de supervivencia}

En los estudios de supervivencia y cuando se valoran los resultados por persona-tiempo hay que tener en cuenta la frecuencia del evento de estudio a la hora de decidir el método de cálculo de la reducción del riesgo que, a su vez, utilizaremos para calcular el NNT. Esto debe hacerse aunque el riesgo sea constante y el periodo de seguimiento de los participantes sea homogéneo.

Vamos a ver cómo se calcularía el NNT con un ejemplo que nos vamos a inventar sobre la marcha. Imaginad que realizamos el estudio y observamos 10 casos de muerte por 100 personas-año en el grupo de intervención y 5 casos por 100 personas-año en el de control.

Suponiendo que los tiempos de supervivencia se distribuyen de forma exponencial, en primer lugar calculamos las proporciones o riesgos acumulados en el grupo de intervención $\left(R_{i}\right)$ y en el control $\left(R_{c}\right)$ :

$\mathrm{R}_{\mathrm{c}}=1-\mathrm{e}^{-10 / 100}=0,095$

$\mathrm{R}_{\mathrm{i}}=1-\mathrm{e}^{-5 / 100}=0,048$ 
Ahora ya podemos calcular el NNT como el inverso de la diferencia de riesgos, tal como ya conocemos:

$\mathrm{NNT}=1 /(0,095-0,048)=21,2$

El error frecuente en esta situación es utilizar directamente el número de eventos según la siguiente fórmula:

NNT $=$ personas-tiempo / (diferencia de eventos)

Si lo aplicamos al caso anterior, quedaría así:

$\mathrm{NNT}=100 /(10-5)=20$

Como veis, el primer método, que es el más adecuado, es algo más conservador y da unos valores más elevados del NNT.

Solo en los casos en los que la frecuencia del suceso es muy baja podremos estimar el NNT utilizando directamente el número de eventos observados. Imaginad que, en el ejemplo anterior, observamos 5 muertes en el grupo de intervención y una en el de control. Podríamos calcular el NNT de la siguiente manera:

$\mathrm{NNT}=100 /(5-1)=25$

De todas formas, no te dejes llevar por el camino más fácil. Solo será correcto calcular el NNT sin la conversión previa de las proporciones acumuladas cuando el número de eventos tenga una frecuencia muy baja $\mathrm{y}$, además, las diferencias de riesgo entre los dos grupos se mantengan proporcionales a lo largo del tiempo. Ante la duda, haz la conversión.

\section{Resumiendo}

Para recapitular un poco todo lo que hemos dicho, podemos recomendar tomar una serie de precauciones para hacer un uso adecuado del NNT y poder interpretarlo de manera correcta.

En primer lugar, no utilices nunca el NNT sin especificar el tratamiento alternativo, la dirección del efecto y la duración del periodo de seguimiento.

Segundo, calcula siempre su intervalo de confianza. En caso de que tenga valores negativos, considera la naturaleza dual de beneficio y daño para tratar de hacer una interpretación más comprensible.

Tercero, no huyas de los decimales. Recuerda que se trata de una estimación más. No debes tener problema en valorar su estimación puntual (aunque no sea un número entero) y su intervalo de confianza.

Por último, comprueba que utilizas la metodología correcta en situaciones más complejas, como aquellas en las que pueda haber implicados factores de confusión $\mathrm{o}$ en los estudios de supervivencia.

Si lo utilizamos con sabiduría, el NNT podrá continuar sus aventuras por su madurez y acompañarnos por lo menos otros 30 años más.

\section{Nos vamos}

Y aquí lo vamos a dejar por hoy.

Ya hemos visto la utilidad del NNT para valorar la eficacia de una intervención. Por ejemplo, nos dice cuántas muertes podemos evitar durante el seguimiento del estudio y que se hubiesen producido si no hubiésemos intervenido.

Pero, ¿qué pasa con los que no se mueren? ¿Hay participantes que se morirán igual si damos que si no damos el tratamiento? Pues el NNT no nos dice nada de eso. Para estudiar este aspecto, 
que mejoraría la valoración del NNT, necesitamos recurrir a otro parámetro: el número restante en riesgo. Pero esa es otra historia...

\section{Bibliografía}

- Laupacis A, Sackett DL, Roberts RS. An assessment of clinically useful measures of the consequences of treatment. $\mathrm{N}$ Engl $\mathrm{J}$ Med.1988;318:1728e33. (NEJM)

- Stang A, Poole C, Bender R. Common problems related to the use of number needed to treat. J Clin Epidemiol.2010;63:820-25. (HTML)

Correspondencia al autor

Manuel Molina Arias

mma1961@gmail.com

Servicio de Gastroenterología.

Hospital Infantil Universitario La Paz.

Madrid. España.

Aceptado para el blog en marzo de 2021 